\title{
The cosmic microwave background anisotropy power spectrum measured by Archeops
}

A. Benoit ${ }^{1}$, P. Ade ${ }^{2}$, A. Amblard ${ }^{3,24}$, R. Ansari ${ }^{4}$, É. Aubourg ${ }^{5,24}$, S. Bargot ${ }^{4}$, J. G. Bartlett ${ }^{3,24}$, J.-Ph. Bernard $^{7,16}$, R. S. Bhatia ${ }^{8}$, A. Blanchard ${ }^{6}$, J. J. Bock ${ }^{8,9}$, A. Boscaleri ${ }^{10}$, F. R. Bouchet ${ }^{11}$, A. Bourrachot ${ }^{4}$, P. Camus ${ }^{1}$, F. Couchot ${ }^{4}$, P. de Bernardis ${ }^{12}$, J. Delabrouille ${ }^{3,24}$, F.-X. Désert ${ }^{13}$, O. Doré ${ }^{11}$, M. Douspis ${ }^{6,14}$, L. Dumoulin ${ }^{15}$, X. Dupac ${ }^{16}$, P. Filliatre ${ }^{17}$, P. Fosalba ${ }^{11}$, K. Ganga ${ }^{18}$, F. Gannaway ${ }^{2}$, B. Gautier ${ }^{1}$, M. Giard ${ }^{16}$, Y. Giraud-Héraud ${ }^{3,24}$, R. Gispert ${ }^{7 \dagger \star}$, L. Guglielmi ${ }^{3,24}$, J.-Ch. Hamilton ${ }^{3,17}$, S. Hanany ${ }^{19}$, S. Henrot-Versillé ${ }^{4}$, J. Kaplan ${ }^{3,24}$, G. Lagache ${ }^{7}$, J.-M. Lamarre ${ }^{7,25}$, A. E. Lange ${ }^{8}$, J. F. Macías-Pérez ${ }^{17}$, K. Madet ${ }^{1}$, B. Maffei ${ }^{2}$, Ch. Magneville ${ }^{5,24}$, D. P. Marrone ${ }^{19}$, S. Masi ${ }^{12}$, F. Mayet ${ }^{5}$, A. Murphy ${ }^{20}$, F. Naraghi ${ }^{17}$, F. Nati ${ }^{12}$, G. Patanchon ${ }^{3,24}$, G. Perrin ${ }^{17}$, M. Piat ${ }^{7}$, N. Ponthieu ${ }^{17}$, S. Prunet ${ }^{11}$, J.-L. Puget ${ }^{7}$, C. Renault ${ }^{17}$, C. Rosset ${ }^{3,24}$, D. Santos ${ }^{17}$, A. Starobinsky ${ }^{21}$, I. Strukov ${ }^{22}$, R. V. Sudiwala ${ }^{2}$, R. Teyssier ${ }^{11,23}$, M. Tristram ${ }^{17}$, C. Tucker ${ }^{2}$, J.-C. Vanel ${ }^{3,24}$, D. Vibert ${ }^{11}$, E. Wakui ${ }^{2}$, and D. Yvon ${ }^{5,24}$

${ }^{1}$ Centre de Recherche sur les Très Basses Températures, BP 166, 38042 Grenoble Cedex 9, France

2 Cardiff University, Physics Department, PO Box 913, 5 The Parade, Cardiff, CF24 3YB, UK

${ }_{3}^{3}$ Physique Corpusculaire et Cosmologie, Collège de France, 11 Pl. M. Berthelot, 75231 Paris Cedex 5, France

4 Laboratoire de l'Accélérateur Linéaire, BP 34, Campus Orsay, 91898 Orsay Cedex, France

5 CEA-CE Saclay, DAPNIA, Service de Physique des Particules, Bât. 141, 91191 Gif sur Yvette Cedex, France

${ }^{6}$ Laboratoire d'Astrophysique de l'Obs. Midi-Pyrénées, 14 avenue E. Belin, 31400 Toulouse, France

7 Institut d'Astrophysique Spatiale, Bât. 121, Université Paris XI, 91405 Orsay Cedex, France

8 California Institute of Technology, 105-24 Caltech, 1201 East California Blvd, Pasadena CA 91125, USA

9 Jet Propulsion Laboratory, 4800 Oak Grove Drive, Pasadena, California 91109, USA

10 IROE-CNR, via Panciatichi, 64, 50127 Firenze, Italy

11 Institut d'Astrophysique de Paris, 98bis boulevard Arago, 75014 Paris, France

12 Gruppo di Cosmologia Sperimentale, Dipart. di Fisica, Univ. "La Sapienza”, P. A. Moro, 2, 00185 Roma, Italy

13 Laboratoire d'Astrophysique, Obs. de Grenoble, BP 53, 38041 Grenoble Cedex 9, France

14 Nuclear and Astrophysics Laboratory, Keble Road, Oxford, OX1 3RH, UK

15 CSNSM-IN2P3, Bât. 108, Campus Orsay, 91405 Orsay Cedex, France

16 Centre d'Étude Spatiale des Rayonnements, BP 4346, 31028 Toulouse Cedex 4, France

17 Institut des Sciences Nucléaires, 53 avenue des Martyrs, 38026 Grenoble Cedex, France

18 Infrared Processing and Analysis Center, Caltech, 770 South Wilson Avenue, Pasadena, CA 91125, USA

19 School of Physics and Astronomy, 116 Church St. S.E., University of Minnesota, Minneapolis MN 55455, USA

20 Experimental Physics, National University of Ireland, Maynooth, Ireland

${ }^{21}$ Landau Institute for Theoretical Physics, 119334 Moscow, Russia

22 Space Research Institute, Profsoyuznaya St. 84/32, Moscow, Russia

23 CEA-CE Saclay, DAPNIA, Service d'Astrophysique, Bât. 709, 91191 Gif sur Yvette Cedex, France

${ }^{24}$ Fédération de Recherche APC, Université Paris 7, Paris, France

25 LERMA, Observatoire de Paris, 61 Av. de l'Observatoire, 75014 Paris, France

Received 16 October 2002 / Accepted 15 December 2002

Send offprint requests to: A. Benoît, e-mail: benoit@grenoble.cnrs.fr

* Richard Gispert passed away few weeks after his return from the early mission to Trapani. 
Abstract. We present a determination by the Archeops experiment of the angular power spectrum of the cosmic microwave background anisotropy in 16 bins over the multipole range $\ell=15-350$. Archeops was conceived as a precursor of the Planck HFI instrument by using the same optical design and the same technology for the detectors and their cooling. Archeops is a balloon-borne instrument consisting of a $1.5 \mathrm{~m}$ aperture diameter telescope and an array of 21 photometers maintained at $\sim 100 \mathrm{mK}$ that are operating in 4 frequency bands centered at 143 , 217, 353 and $545 \mathrm{GHz}$. The data were taken during the Arctic night of February 7, 2002 after the instrument was launched by CNES from Esrange base (Sweden). The entire data cover $\sim 30 \%$ of the sky. This first analysis was obtained with a small subset of the dataset using the most sensitive photometer in each CMB band (143 and $217 \mathrm{GHz})$ and $12.6 \%$ of the sky at galactic latitudes above 30 degrees where the foreground contamination is measured to be negligible. The large sky coverage and medium resolution (better than 15 arcmin) provide for the first time a high signal-to-noise ratio determination of the power spectrum over angular scales that include both the first acoustic peak and scales probed by COBE/DMR. With a binning of $\Delta \ell=7$ to 25 the error bars are dominated by sample variance for $\ell$ below 200. A companion paper details the cosmological implications.

Key words. cosmic microwave background - cosmology: observations - submillimeter

\section{Introduction}

Observations of the Cosmic Microwave Background (CMB) temperature anisotropies have provided answers to fundamental questions in cosmology. The observational determination of the CMB angular power spectrum has already led to important insights on the structure and evolution of the universe. Most notable are the conclusions that the geometry of space is essentially flat (Miller et al. 1999; de Bernardis et al. 2000; Hanany et al. 2000) and that the measurements are consistent with the inflationary paradigm (Netterfield et al. 2002; Lee et al. 2001; Halverson et al. 2002; Sievers et al. 2002; Rubiño-Martin et al. 2002). Since the first detection of CMB anisotropy with COBE/DMR (Smoot et al. 1992), a host of experiments have measured the spectrum down to sub-degree scales, but measurements at large angular scales remain difficult, due to the large sky coverage required to access these modes. This difficulty will be overcome by the future full-sky space missions MAP and Planck.

This paper presents the first results from Archeops, an experiment designed to obtain large sky coverage in a single balloon flight. A detailed description of the instrument inflight performance will be given in Benoît et al. (2003b); here we provide only essential information. Archeops ${ }^{1}$ is a balloon-borne experiment with a $1.5 \mathrm{~m}$ off-axis Gregorian telescope and a bolometric array of 21 photometers operating at frequency bands centered at $143 \mathrm{GHz}$ (8 bolometers), $217 \mathrm{GHz}$ (6), $353 \mathrm{GHz}$ ( $6=3$ polarized pairs $)$ and $545 \mathrm{GHz}$ (1). The focal plane is maintained at a temperature of $\sim 100 \mathrm{mK}$ using a ${ }^{3} \mathrm{He}-$ ${ }^{4} \mathrm{He}$ dilution cryostat. Observations are carried out by turning the payload at $2 \mathrm{rpm}$ producing circular scans at a fixed elevation of $\sim 41 \mathrm{deg}$. Observations of a single night cover a large fraction of the sky as the circular scans drift across the sky due to the rotation of the Earth.

\section{Observations and processing of the data}

The experiment was launched on February 7, 2002 by the $\mathrm{CNES}^{2}$ from the Swedish balloon base in Esrange, near Kiruna, Sweden, $68^{\circ} \mathrm{N}, 20^{\circ} \mathrm{E}$. It reached a float altitude of $\sim 34 \mathrm{~km}$ and landed 21.5 hours later in Siberia near Noril'sk, where it was recovered by a Franco-Russian team. The night-time scientific

\footnotetext{
${ }^{1}$ See http://www . archeops.org

2 Centre National d'Études Spatiales, the French national space agency.
}

observations span 11.7 hours of integration from $15.3 \mathrm{UT}$ to $3.0 \mathrm{UT}$ the next day. Figure 1 shows the Northern galactic part of the sky observed during the flight.

A detailed description of the data processing pipeline will be given in Benoît et al. (2003c). Pointing reconstruction, good to 1 arcmin, is performed using data from a bore-sight mounted optical star sensor aligned to each photometer using Jupiter observations. The raw Time Ordered Information (TOI), sampled at $153 \mathrm{~Hz}$, are preprocessed to account for the readout electronics and response variations. Corrupted data (including glitches), representing less than $1.5 \%$, are flagged. Low frequency drifts correlated to various templates (altitude, attitude, temperatures, CMB dipole) are removed from the data. To remove residual dust and atmospheric signal, the data are decorrelated with the high frequency photometers and a synthetic dust timeline (Schlegel et al. 1998).

The CMB dipole is the prime calibrator of the instrument. The absolute calibration error against the dipole measured by COBE/DMR (Fixsen et al. 1994) is estimated to be less than $4 \%($ resp. $8 \%$ ) in temperature at $143 \mathrm{GHz}$ (resp. $217 \mathrm{GHz}$ ). Two other independent calibration methods, both with intrinsic uncertainty of $\sim 10 \%$, give responsivities relative to the dipole calibration at 143 (resp. $217 \mathrm{GHz}$ ) of -5 (resp. $+6 \%$ ) on Jupiter and -20 (resp. $-5 \%$ ) with COBE-FIRAS Galactic Plane emission.

The beam shapes of the photometers measured on Jupiter are moderately elliptical, having a ratio of the major to minor axis of 1.2 (resp. 1.5) at $143 \mathrm{GHz}$ (resp. $217 \mathrm{GHz}$ ), and have an equivalent $F W H M$ of 11 arcmin (resp. 13). The error in beam size is less than $10 \%$. The effective beam transfer function for each photometer, determined with simulations, is taken into account in the analysis and is in excellent agreement with analytical estimates (Fosalba et al. 2002).

\section{Analysis}

In this paper, we use data from only a single detector at each of the CMB frequencies, 143 and $217 \mathrm{GHz}$, with a sensitivity of 90 and $150 \mu \mathrm{K}_{\mathrm{CMB}} \cdot \mathrm{s}^{1 / 2}$ respectively. To avoid the necessity of detailed modelling of Galactic foregrounds, we restrict the sky coverage to $b>+30 \mathrm{deg}$, giving a total of $\sim 100000$ 15 arcmin pixels (HEALPIX nside $=256$ ) covering $12.6 \%$ of the sky (see Fig. 1). To extract the CMB power spectrum, we use the MASTER analysis methodology (Hivon et al. 2002), 


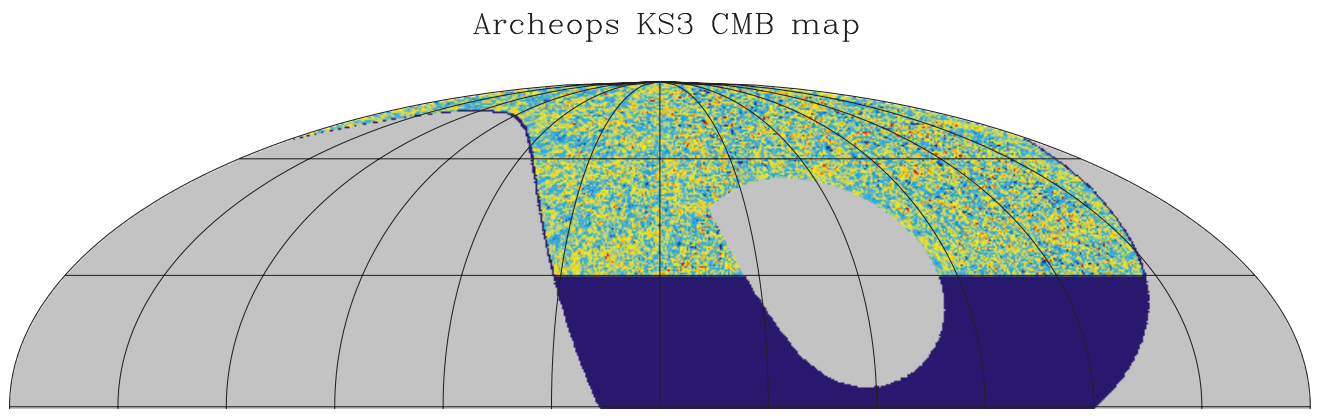

Fig. 1. Archeops CMB map (Galactic coordinates, centered on the Galactic anticenter, Northern hemisphere) in HEALPIX pixelisation (Gorski et al. 1998) with 15 arcmin pixels and a 15 arcmin Gaussian smoothing. The map is a two-photometers coaddition. The dark blue region is not included in the present analysis because of possible contamination by dust. The colors in the map range from -500 to $500 \mu \mathrm{K}_{\mathrm{CMB}}$.

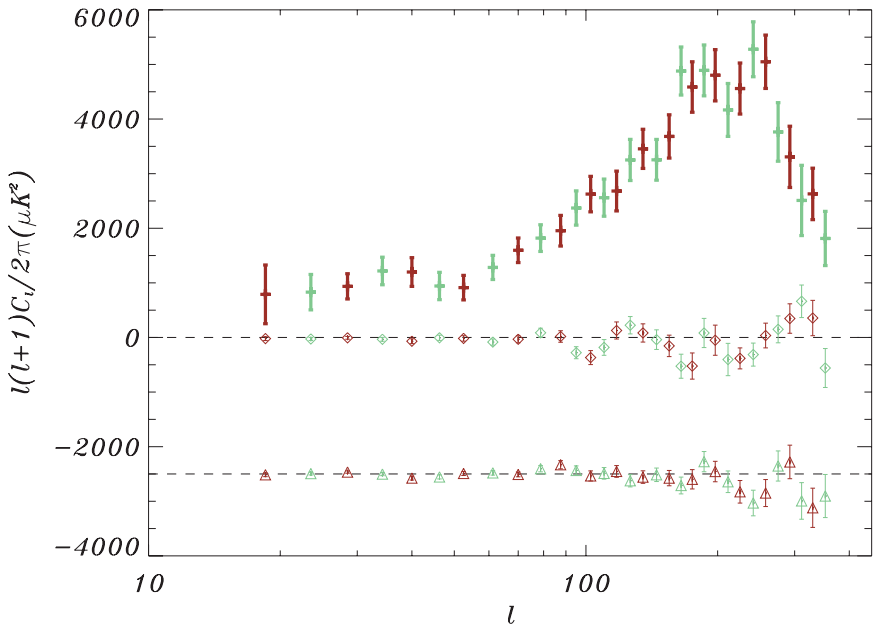

Fig. 2. The Archeops CMB power spectrum for the combination of the two photometers. Green and red data points correspond to two overlapping binnings and are therefore not independent. The light open diamonds show the null test resulting from the self difference $(S D)$ of both photometers and the light open triangles correspond to the difference $(D)$ of both photometers (shifted by $-2500 \mu \mathrm{K}^{2}$ for clarity) as described in Sect. 4 and shown in Table 1.

which achieves speed by employing sub-optimal (but unbiased) map-making and spectral determinations.

First, the Fourier noise power spectrum is estimated for each photometer. Signal contamination is avoided by subtracting the data projected onto a map (and then re-read with the scanning strategy) from the initial TOI. This raw noise power spectrum is then corrected for two important effects (Benoît et al. 2003d): (i) pixelisation of the Galactic signal that leads to an overestimate of the noise power spectrum: sub-pixel frequencies of the signal are not subtracted from the inital TOI leaving extra signal at high frequency; (ii) due to the finite number of samples per pixel, noise remains in the map and is subtracted from the initial TOI, inducing an underestimation of the actual noise in the final TOI (Ferreira \& Jaffe 2000; Stompor et al. 2002). Simulations, including realistic noise, Galactic dust and CMB anisotropies, indicate that both corrections are independent of the shape of the true noise power spectrum, and thus permit an unbiased estimate of the latter with an accuracy better than $1 \%$ at all frequencies. The corresponding uncertainty in the noise power spectrum estimation is included in the error bars of the $C_{\ell}$ spectrum.

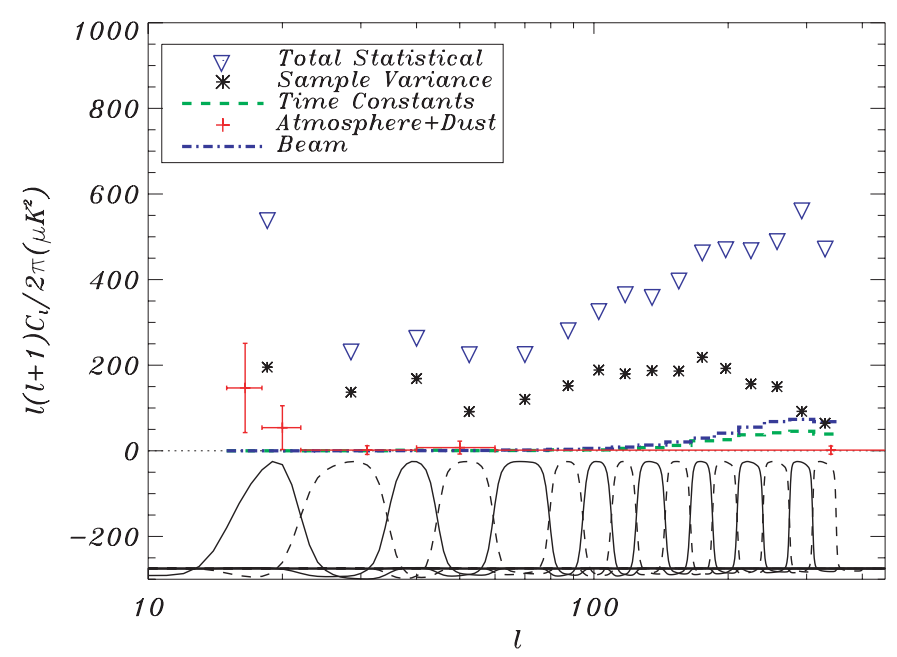

Fig. 3. Contamination by systematics: the Archeops CMB power spectrum statistical error bars (including noise and sample variance) are shown as the blue triangles. The large error bar in the first bin mainly comes from the high-pass filtering. A conservative upper-limit to contamination by dust and atmospheric signal is shown in red crosses, with a $\ell$ different binning to enhance the low $\ell$ side. Beam and time constants uncertainties are shown in dot-dashed blue and dashed green (see text). The $7 \%$ temperature calibration uncertainty is not shown here. The window functions are shown at the bottom of the figure.

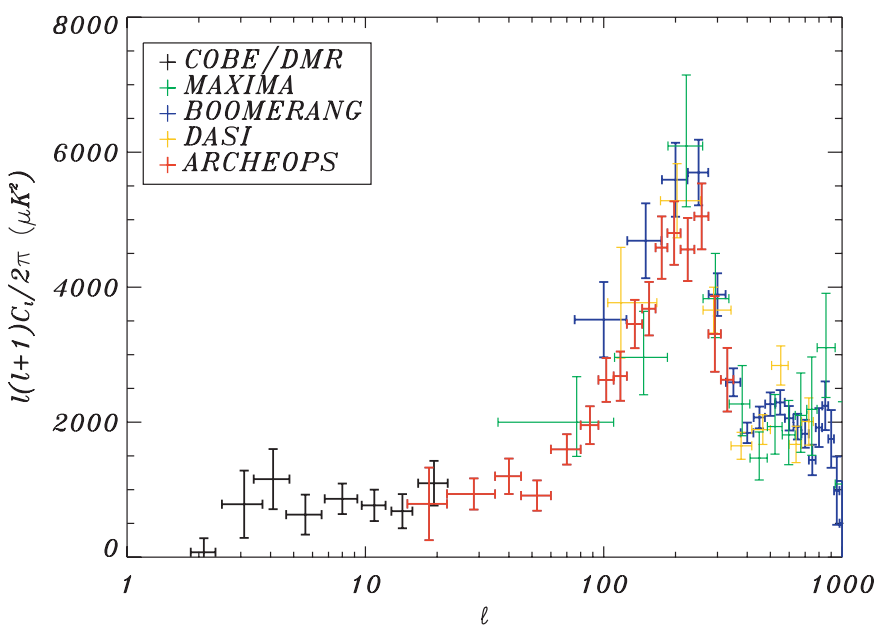

Fig. 4. The Archeops power spectrum compared with results of COBE, Boomerang, Dasi, Maxima (Tegmark 1996; Netterfield et al. 2002; Lee et al. 2001; Halverson et al. 2002). 
We construct maps by bandpassing the data between 0.3 and $45 \mathrm{~Hz}$, corresponding to about $30 \mathrm{deg}$ and 15 arcmin scales, respectively. The high-pass filter removes remaining atmospheric and galactic contamination, the low-pass filter suppresses non-stationary high frequency noise. The filtering is done in such a way that ringing effects of the signal on bright compact sources (mainly the Galactic plane) are smaller than $\sim 36 \mu \mathrm{K}^{2}$ on the CMB power spectrum in the very first $\ell-$ bin, and negligible for larger multipoles. Filtered TOI of each absolutely calibrated detector are co-added on the sky to form detector maps. The bias of the CMB power spectrum due to filtering is accounted for in the MASTER process through the transfer function. The map shown in Fig. 1 is obtained by combining the maps of each of the photometers. A $1 / \sigma^{2}$ weighting of the data was done in each pixel, where $\sigma^{2}$ is the variance of the data in that pixel. This map shows significant extra variance compared to the difference map on degree angular scales which is attributed to sky-stationary signal.

We estimate the CMB power spectrum in 16 bins ranging from $\ell=15$ to $\ell=350$. The window functions derived from the multipole binning and renormalized to equal amplitude for clarity are shown at the bottom of Fig. 3. They are nearly top-hat functions due to the large sky coverage. The bins can therefore be approximated as independent: off-diagonal terms in the covariance matrix are less than $\sim 12 \%$. For the purpose of estimating the power spectrum we made a map that combines the data of the two photometers using two different weighting techniques. Up to $\ell=310$ the data of each photometer has equal weight and at larger $\ell$ values the data is noise weighted. This is valid because the multipole bins are nearly independent. It is also advantageous because it minimizes the overall statistical noise over the entire $\ell$ spectrum; equal weighting gives smaller error bars at small $\ell$ and noise weighting gives smaller error bars at large $\ell$.

\section{Results and consistency tests}

The Archeops power spectrum is presented in Fig. 2 and in Table 1. Two different binnings corresponding to overlapping, shifted window functions (therefore not independent) were used. Archeops provides the highest $\ell$ resolution up to $\ell=$ 200 ( $\Delta \ell$ from 7 to 25 ) and most precise measurement of the angular power spectrum for $15<\ell<300$ to date. Samplevariance contributes $50 \%$ or more of the total statistical error up to $\ell \sim 200$.

The Archeops scanning strategy (large circles on the sky) provides a robust test of systematic errors and data analysis procedures: by changing the sign of the filtered TOIs every other circle, a TOI that should not contain any signal is obtained once it is projected on the sky. This TOI has the same noise power spectrum as the original one. This null test is referred to as the self-difference $(S D)$ test. The angular power spectrum of such a dataset should be consistent with zero at all multipoles because successive circles largely overlap. This test has been performed with the two photometers independently. The spectra are consistent with zero at all modes: $\chi^{2} /$ ndf of $21 / 16$ (resp. 27/16) at $143 \mathrm{GHz}$ (resp. $217 \mathrm{GHz}$ ). Performed on the two-photometers co-added map, the same test gives a power spectrum consistent with zero, with a $\chi^{2} /$ ndf of $25 / 16$ (see
Table 1. The Archeops CMB power spectrum for the best two photometers (third column). Data points given in this table correspond to the red points in Fig. 2. The fourth column shows the power spectrum for the self difference $(S D)$ of the two photometers as described in Sect. 4. The fifth column shows the power spectrum for the difference $(D)$ between the two photometers.

\begin{tabular}{rrrrr}
\hline \hline$\ell_{\min }$ & $\ell_{\max }$ & $\frac{\ell(\ell+1) C_{\ell}}{(2 \pi)}(\mu \mathrm{K})^{2}$ & \multicolumn{1}{c}{$S D(\mu \mathrm{K})^{2}$} & \multicolumn{1}{c}{$D(\mu \mathrm{K})^{2}$} \\
\hline 15 & 22 & $789 \pm 537$ & $-21 \pm 34$ & $-14 \pm 34$ \\
22 & 35 & $936 \pm 230$ & $-6 \pm 25$ & $34 \pm 21$ \\
35 & 45 & $1198 \pm 262$ & $-69 \pm 45$ & $-75 \pm 35$ \\
45 & 60 & $912 \pm 224$ & $-18 \pm 50$ & $9 \pm 37$ \\
60 & 80 & $1596 \pm 224$ & $-33 \pm 63$ & $-8 \pm 44$ \\
80 & 95 & $1954 \pm 280$ & $17 \pm 105$ & $169 \pm 75$ \\
95 & 110 & $2625 \pm 325$ & $-368 \pm 128$ & $-35 \pm 92$ \\
110 & 125 & $2681 \pm 364$ & $127 \pm 156$ & $46 \pm 107$ \\
125 & 145 & $3454 \pm 358$ & $82 \pm 166$ & $-57 \pm 114$ \\
145 & 165 & $3681 \pm 396$ & $-154 \pm 196$ & $-75 \pm 140$ \\
165 & 185 & $4586 \pm 462$ & $-523 \pm 239$ & $-97 \pm 177$ \\
185 & 210 & $4801 \pm 469$ & $-50 \pm 276$ & $44 \pm 187$ \\
210 & 240 & $4559 \pm 467$ & $-382 \pm 192$ & $-326 \pm 206$ \\
240 & 275 & $5049 \pm 488$ & $35 \pm 226$ & $-349 \pm 247$ \\
275 & 310 & $3307 \pm 560$ & $346 \pm 269$ & $220 \pm 306$ \\
310 & 350 & $2629 \pm 471$ & $356 \pm 323$ & $-619 \pm 358$ \\
\hline
\end{tabular}

Fig. 2). These results show that there is no significant correlated noise among the two photometers and that the noise model is correct. They limit the magnitude of non-sky-stationary signals to a small fraction of the sky-stationary signal detected in the maps.

A series of Jack-knife tests shows agreement between the first and second halves of the flight (the difference of the power spectra has $\chi^{2} /$ ndf $\left.=21 / 16\right)$, left and right halves of the map obtained with a cut in Galactic longitude $\left(\chi^{2} /\right.$ ndf $\left.=15 / 16\right)$. Individual power spectra of the two photometers agree once absolute calibration uncertainties are taken into account. The power spectrum measured on the differences $(D)$ between the two photometers is consistent with zero with a $\chi^{2} /$ ndf of $22 / 16$ (Fig. 2) showing that the electromagnetic spectrum of the skystationary signal is consistent with that of the CMB. The measured $\mathrm{CMB}$ power spectrum depends neither on the Galactic cut (20,30 and 40 degrees north from the Galactic plane), nor on the resolution of the maps $(27,14$ and 7 ' pixel size) nor on the TOI high-pass filtering frequencies $(0.3,1$ and $2 \mathrm{~Hz})$.

Several systematic effects have been estimated and are summarized in Fig. 3, along with the statistical errors (blue triangles). The high frequency photometer $(545 \mathrm{GHz})$ is only sensitive to dust and atmospheric emission, and thus offers a way to estimate the effect of any residual Galactic or atmospheric emission. Extrapolation of its power spectrum using a Rayleigh-Jeans spectrum times a $v^{2}$ emissivity law between 545 and $217 \mathrm{GHz}$ and as $v^{0}$ between 217 and $143 \mathrm{GHz}$ gives an upper-limit on the possible contamination by atmosphere (dominant) and dust. The combination of both is assumed to be much less than $50 \%$ of the initial contamination after the decorrelation process. The subsequent conservative upper-limit for dust and atmosphere contamination is shown in red crosses in Fig. 3. The contamination appears negligible in all bins but the first one ( $\ell=15$ to 22 ). High frequency spectral 
leaks in the filters at 143 and $217 \mathrm{GHz}$ were measured to give a contribution less than half of the above contamination. In the region used to estimate the $\mathrm{CMB}$ power spectrum there are 651 extragalactic sources in the Parkes-MIT-NRAO catalog. These sources are mainly AGN, and their flux decreases with frequency. We have estimated their contribution to the power spectrum using the WOMBAT tools (Sokasian et al. 2001). At 143 (resp. 217) $\mathrm{GHz}$ this is less than 2 (resp. 1) percent of the measured power spectrum at $\ell \sim 350$. The beam and photometer time constant uncertainties were obtained through a simultaneous fit on Jupiter crossings. Their effect is shown as the dot-dashed blue and green-dashed lines in Fig. 3. The beam uncertainty includes the imperfect knowledge of the beam transfer function for each photometer's elliptical beam. Beam and time constants uncertainties act as a global multiplicative factor, but in the figure we show the $1 \sigma$ effect on a theoretical power spectrum that has a good fit to the data. After the coaddition of the two photometers, the absolute calibration uncertainty (not represented in Fig. 3) is estimated as 7\% (in CMB temperature units) with Monte-Carlo simulations.

As a final consistency test, the Archeops $C_{\ell}$ are computed using two additional independent methods. The first is based on noise estimation with an iterative multi-grid method, MAPCUMBA (Doré et al. 2001), simple map-making and $C_{\ell}$ estimation using SpICE (Szapudi et al. 2001) which corrects for mask effects and noise ponderation through a correlation function analysis. The second is based on MIRAGE iterative map-making (Yvon et al. 2003) followed by multi-component spectral matching (Cardoso et al. 2002; Patanchon et al. 2003; Delabrouille et al. 2002). All methods use a different mapmaking and $C_{\ell}$ estimation. Results between the three methods agree within less than one $\sigma$. This gives confidence in both the $C_{\ell}$ and in the upper-limits for possible systematic errors. Table 1 provides the angular power spectrum which is used for cosmological parameter extraction (Benoît et al. 2003a).

A comparison of the present results with other recent experiment and COBE/DMR is shown in Fig. 4. There is good agreement with other experiments, given calibration uncertainties, and particularly with the power COBE/DMR measures at low $\ell$ and the location of the first acoustic peak. Work is in progress to improve the intercalibration of the photometers, the accuracy and the $\ell$ range of the power spectrum: the low $\ell$ range will be improved increasing the effective sky area for $\mathrm{CMB}$ (which requires an efficient control of dust contamination), the high $\ell$ range will be improved by including more photometer pixels in the analysis.

\section{Conclusions}

The Archeops experiment has observed a large portion of the sky. Maps from the two highest sensitivity detectors at 143 and $217 \mathrm{GHz}$ show consistent, sky-stationary anisotropy signal that appears inconsistent with any known astrophysical source other than CMB anisotropy. The angular power spectrum of this signal at multipoles between $\ell=15$ and $\ell=350$ shows a clear peak at $\ell \simeq 200$. These results are consistent with predictions by inflationary-motivated cosmologies. Archeops provides the highest signal-to-noise ratio mapping of the first acoustic peak and its low $-\ell$ side of any experiment to date and covers the largest number of decades in $\ell$. It has been obtained with a limited integration time (half a day) using a technology similar to that of the Planck HFI experiment. An extensive set of tests limits the contribution of systematic errors to a small fraction of the statistical and overall calibration errors in the experiment. More data reduction is under way to increase the accuracy and $\ell$ range of the power spectrum. The determination of cosmological parameters are discussed in a companion paper (Benoît et al. 2003a).

Acknowledgements. The authors would like to thank the following institutes for funding and balloon launching capabilities: CNES (French space agency), PNC (French Cosmology Program), ASI (Italian Space Agency), PPARC, NASA, the University of Minnesota, the American Astronomical Society and a CMBNet Research Fellowship from the European Commission. Healpix package was used throughout the data analysis (1998).

\section{References}

Benoît, A., Ade, P., Amblard, A., et al. 2002a, Astropart. Phys., 17, 101

Benoît, A., Ade, P., Amblard, A., et al. 2003a, A\&A, 399, L25

Benoît, A., Ade, P., Amblard, A., et al. 2003b, in preparation

Benoît, A., Ade, P., Amblard, A., et al. 2003c, in preparation

Benoît, A., Ade, P., Amblard, A., et al. 2003d, in preparation

de Bernardis, P., Ade, P. A. R., Bock, J. J., et al. 2000, Nature, 404, 955

Cardoso, J. F., Snoussi, H., Delabrouille, J., \& Patanchon, G. 2002, Proc. EUSIPCO02 Conf., Toulouse, Sep. 2002 [astro-ph/0209466]

Delabrouille, J., Cardoso, J. F., \& Patanchon, G. 2002, MNRAS, submitted [astro-ph/0211504]

Doré, O., Teyssier, R., Bouchet, F. R., Vibert, D., \& Prunet, S. 2001, A\&A, 374, 358

Ferreira, P. G., \& Jaffe, A. H. 2000, MNRAS, 312, 89

Fixsen, P. J., Cheng, E. S., Cottingham, D. A., et al. 1994, ApJ, 420, 445

Fosalba, P., Doré, O., \& Bouchet, F. R. 2002, Phys. Rev. D, 65, 63003

Gorski, K. M., Hivon, E., \& Wandelt, B. D. 1998, Proc. of the MPA/ESO Conf. on Evolution of Large-Scale Structure: from Recombination to Garching, 2-7 August 1998, ed. A. J. Banday, R. K. Sheth, \& L. Da Costa [astro-ph/9812350]

Halverson, N. W., Leitch, E. M., Pryke, C., et al. 2002, ApJ, 568, 38

Hanany, S., Ade, P., Balbi, A., et al. 2000, ApJ, 545, L5

Hivon, E., Gorski, K. M., Netterfield, C. B., et al. 2002, ApJ, 567, 2

Lee, A. T., Ade, P., Balbi, A., et al. 2001, ApJ, 561, L1

Miller, A. D., Caldwell, R., Devlin, M. J., et al. 1999, ApJ, 524, L1

Netterfield, C. B., Ade, P. A. R., Bock, J. J., et al. 2002, ApJ, 571, 604

Patanchon, G., Snoussi, H., Cardoso, J.-F., \& Delabrouille, J. 2003, Proc. of the PSIP03 Conf., Grenoble, January 2003 [astro-ph/0302078]

Rubiño-Martin, J. A., Rebolo, R., Carreira, P., et al. 2002, MNRAS, submitted [astro-ph/0205367]

Schlegel, D., Finkbeiner, D., \& Davis, M. 1998, ApJ, 500, 525

Sievers, J. L., Bond, J. R., Cartwright, J. K., et al. 2002, ApJ, submitted [astro-ph/0205387]

Smoot, G. F., Bennett, C. L., Kogut, A., et al. 1992, ApJ, 396, L1

Sokasian, A., Gawiser, E., \& Smoot, G. F. 2001, ApJ, 562, L88

Stompor, R., Balbi, A., Borrill, J. D., et al. 2002, Phys. Rev. D, 65, 022003

Szapudi, I., Prunet, S., Pogosyan, D., Szalay, A. S., Bond, J. R., et al. 2001, ApJ, 548, L115

Tegmark, M. 1996, ApJ, 464, L35

Yvon, D., et al. 2003, in preparation 